\title{
Complications, imaging results, and midterm clinical outcomes of pipeline embolisation device in the treatment of cerebral aneurysms
}

\author{
Hamed Asadi ${ }^{1}$, Timothy Phillips ${ }^{2}$, Richard Dowling ${ }^{1}$, Bernard Yan ${ }^{1}$, Peter Mitchell ${ }^{1}$ \\ ${ }^{1}$ Melbourne Brain Centre, Department of Medicine, Royal Melbourne Hospital, University of Melbourne, Parkville, Australia \\ ${ }^{2}$ Neurological Intervention \& Imaging Service of Western Australia, Perth, Australia \\ Email: peter.mitchell@mh.org.au
}

Received 10 November 2013; revised 11 December 2013; accepted 17 December 2013

Copyright (c) 2014 Hamed Asadi et al. This is an open access article distributed under the Creative Commons Attribution License, which permits unrestricted use, distribution, and reproduction in any medium, provided the original work is properly cited. In accordance of the Creative Commons Attribution License all Copyrights ¿ 2014 are reserved for SCIRP and the owner of the intellectual property Hamed Asadi et al. All Copyright (C) 2014 are guarded by law and by SCIRP as a guardian.

\section{ABSTRACT}

Background: The introduction of pipeline embolisation device (PED) has improved the feasibility of endovascular treatment of intracranial aneurysms. The device allows for endoluminal reconstruction across the aneurysm neck but is permeable enough that flow is preserved across the pressure gradients into sidebranch arteries. In spite of higher rates of aneurysm occlusion, there is lack of data concerning medium to long-term clinical and imaging results. Methods: This study was a prospective single center analysis of complications, imaging results, and medium term clinical outcomes after PED treatment of intracranial aneurysms. We included cases over a 17-month period in a tertiary interventional neuroradiology center. We collected data on demographics, vascular risk factors, clinical presentation, angiographic results post treatment, angiographic follow-up and clinical follow-up. Results: Thirty-three patients were included, 25 females and 8 males, with mean age of 55 years; 3 presented with acute subarachnoid hemorrhage and 30 for elective treatment. Thirty-seven aneurysms were treated: 35 Internal Carotid Artery (ICA), 1 basilar trunk fusiform, and 1 vertebral artery intradural dissecting aneurysms. No deaths have occurred. Five patients suffered transient neurological complications (15\%). Overall aneurysm occlusion was demonstrated in $85 \%$ of patients at the end of one year. Discussion: Overall, the technique of flow diversion and endoluminal reconstruction differs greatly from the established endosaccular packing techniques of standard coiling, balloon remodeling, or stent assisted coiling. Our midterm follow-up confirms that, the rates of clinically significant complications compare favorably with published data on stent assisted coiling, potentially making these devices a truly revolutionizing technique.

\section{KEYWORDS}

Cerebral Aneurysm; Flow Diverting Stents; Pipline Embolisation Device; Endovascular Treatment

\section{INTRODUCTION}

The introduction of flow-diverting or flow-directing devices is a significant addition to the endovascular treatment of intracranial aneurysms. These stent-like devices are deployed in the parent artery to treat aneurysms by endoluminal reconstruction across the aneurysm neck, with or without endosaccular coil embolization. The mesh wall of the device is dense enough to disrupt flow in and out of the aneurysm sac leading to stasis and thrombosis; but permeable enough that flow is preserved across the pressure gradients into side-branch arteries. Over a longer period the device acts as scaffolding on which new endothelium forms, thereby reconstructing the parent artery and excluding the aneurysm from the circulation.

The pipeline embolization device (PED, ev3 California) is a self-expanding cylindrical device composed of 48 braided strands of cobalt chromium and platinum, each $28-33 \mu \mathrm{m}$ in calibre. When fully deployed, the device expands to its nominal caliber and shortens by a factor of 2.5 to its nominal length. When unrestrained, a single PED has 30\% - 35\% metal surface area coverage, or porosity. 
Although several large case series have been published, there is a paucity of long-term clinical and imaging follow-up in the literature, in which two of the few published series to date with greater than one year follow-up are of 12 and 18 patients respectively [1,2]. Initially the published data focused on ischemic events, in-construct stenosis, mass effect, and delayed aneurysm rupture [2-4], however more recently a subset of unusual hemorrhagic events after PED have surfaced [5-10].

This study was a prospective single center analysis of complications, imaging results, and long-term clinical outcomes after PED treatment of anterior and posterior circulation aneurysms in 33 patients.

\section{MATERIALS AND METHODS}

\subsection{Patient Selection}

Data was prospectively recorded for all cases where PED was used over a 17-month period in tertiary interventional neuroradiology center. Case selection was made on the basis of aneurysm morphology for which standard balloon-remodeling, stent-assisted, and neurosurgical techniques are not suitable or have been unsuccessful. The decision to treat with PED was by consensus opinion at a multidisciplinary meeting involving interventional neuroradiologists and subspecialist cerebrovascular neurosurgeons.

\subsection{Procedural Methods}

\subsubsection{Antiplatelet Therapy}

$\mathrm{T}$ All patients were pre-medicated with dual antiplatelet therapy. The 30 booked elective cases were prescribed 75 mg Clopidogrel and $100 \mathrm{mg}$ aspirin for 3 days minimum. The 3 acute cases were given $450 \mathrm{mg}$ clopidogrel and $300 \mathrm{mg}$ aspirin 4 - 5 hours before the operation. All cases were prescribed 6 months of $75 \mathrm{mg}$ clopidogrel and lifelong $100 \mathrm{mg}$ aspirin postoperatively.

\subsubsection{Anticoagulation}

In 32 cases the patient was heparinized after the common femoral artery was accessed. In the case of the recently ruptured dissecting blister Internal Carotid Artery (ICA) aneurysm, anticoagulation was delayed until the aneurysm was coiled. All patients were prescribed a heparin infusion for $48 \mathrm{hrs}$ post operatively.

\subsubsection{Neurointerventional Procedure}

All cases were performed under general anesthetic. Standard neurointerventional co-axial or tri-axial access delivery systems were used. A jailed microcatheter technique was employed if endosaccular coiling was being performed. The PED deployment technique has been previously described [3,11].

\subsection{Clinical Follow-Up}

All patients were assessed on multiple occasions by interventional neuroradiologists in outpatient clinic; the minimum number of visits was 3; at 6 - 8 weeks, at 6 months, and at 12 months. Additional clinical follow-up during and after these timeframes were used when clinically indicated. Their neurological outcome was assessed on the modified Rankin Scale.

\subsection{Imaging Follow-Up}

The imaging follow-up regimen varied, depending on the other intracranial devices present, logistical factors (patients in remote areas) and the patient's preferences.

Catheter angiography remains the gold standard, but other non-invasive imaging modalities can also evaluate stent patency and aneurysm occlusion.

CT Angiography (CTA) can demonstrate stent patency and aneurysm occlusion when no endosaccular coils are present. Other authors have also reported this $[12,13]$. Using an edge-enhancing high-spatial-resolution kernel (H60s or H60f) anecdotally results in better delineation of device patency, aneurysm filling, and aneurysm morphology. Use of edge-enhancing high resolution kernels to assess stent patency has been reported in CT coronary angiography [14].

MRI and MR Angiography (MRA) are less affected by coil artifact, can demonstrate endosaccular thrombus, and can exclude aneurysm filling. MRI/MRA is however more limited in imaging the device lumen, and $\mathrm{T} 1$ hyperintense endosaccular clot can occasionally result in the false appearance of blood flow on time-of-flight MRA.

\section{RESULTS}

\subsection{Patient Demographics}

33 consecutive patients (25 females, ratio 3.1:1), with mean age 55 years were treated with PED between September 2009 and February 2011.

\subsection{Clinical Presentations}

\subsubsection{Three Acute Presentations}

Patient 13 was a 49-year-old male who presented with acute and sudden onset left retro-orbital pain, swelling, and double vision. On examination he had cranial nerves III, IV, and VI palsies, chemosis, and proptosis. A catheter cerebral angiogram on day 0 demonstrated a direct left carotid-cavernous fistula secondary to a ruptured cavernous segment ICA aneurysm. After on-table 450 $\mathrm{mg}$ clopidogrel and $300 \mathrm{mg}$ aspirin, two PEDs were used to create a flow-diverting construct across the aneurysm neck. This acutely reduced the fistulous flow on-table. Coil embolization of the cavernous sinus was performed 
on day 0 , day 4, and at 8 months; with complete occlusion of the fistula. At 18 months he had no signs of carotid cavernous fistula (no chemosis, proptosis, venous engorgement or retinal hemorrhages), but persistent double vision. See Figure 1.

Patient 15 was a 30-year-old female who presented with World Federation of Neurosurgery (WFNS) clinical grade 2, Fisher CT grade 2 subarachnoid hemorrhage. No intracranial lesion was detected on three catheter angiograms and MR angiography between days 0 and 7 . Multiple extracranial and extradural carotid and vertebral artery dissections, and fibromuscular dysplasia were observed. Repeat MRI and catheter angiography performed on day 17 and 18 demonstrated a newly apparent left supraclinoid ICA lateral side-wall $3.5 \mathrm{~mm}$ blister aneurysm. On day 29 the aneurysm had enlarged to $5 \mathrm{~mm}$ and was treated with endosaccular coiling and a single PED construct. On-table aneurysm rupture complicated the endosaccular coiling prior to PED deployment. The subarachnoid extravasation resolved without any change in

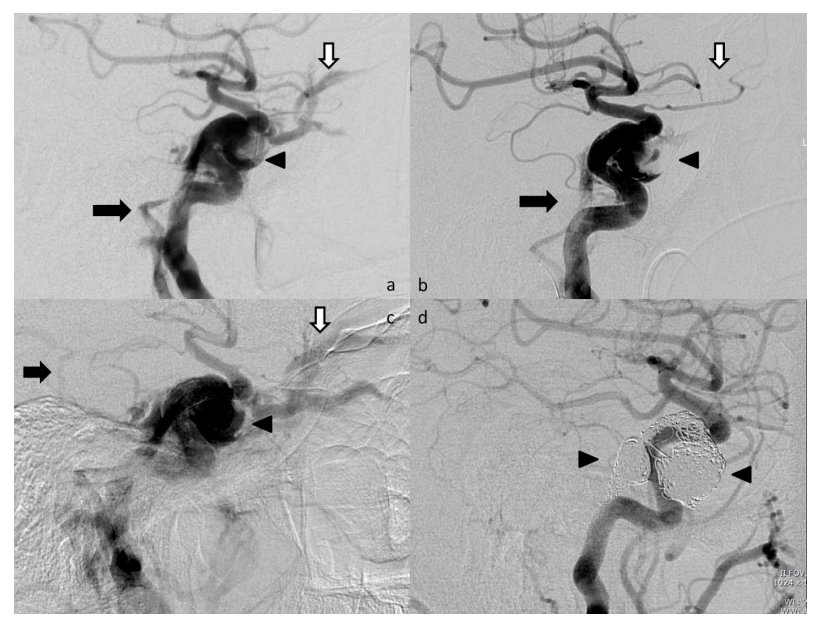

Figure 1. All images are digital subtracted angiograms from a left internal carotid artery injection, during the arterial phase, in Patient 13. a) Day 0 after rupture of a cavernous ICA aneurysm with resultant acute carotid-cavernous fistula. Arrowhead indicating the cavernous aneurysm and the abnormal opacification of the cavernous sinus. Black arrow indicating the abnormal filling of the inferior petrosal sinus, and a white arrow indicating the filling of the superior ophthalmic vein; b) Day 0 after deployment of a flow-diverting construct (3 PEDs) in the cavernous ICA, demonstrating less rapid filling of the cavernous sinus and delayed emptying of the aneurysm (black arrowhead), less rapid filling of the inferior petrosal sinus, and absent filling of the superior ophthalmic vein in the arterial phase; c) Repeat angiography on day 6 demonstrating increased superior ophthalmic vein filling and dilatation (white arrow), expansion and increased filling of the cavernous sinus (black arrowhead), and cortical venous reflux (black arrow); d) Follow-up angiography at 1 year after three further trans-venous cavernous sinus coilings (arrowheads). No abnormal fistulous filling of the cavernous sinus, ophthalmic veins, inferior petrosal sinus, or cortical veins is demonstrated in the arterial phase. clinical parameters, and PED deployment was completed. The patient awoke with a headache post procedure but was otherwise neurologically unchanged. At 6 months she is back at work, mRS 0, and follow-up MRA demonstrates no aneurysm filling.

Patient 29 was a 78-year-old male patient presenting with an acute painful third cranial nerve palsy secondary to an $8 \mathrm{~mm}$ wide necked posterior communicating artery aneurysm. Coiling on day 1 was partially successful, with only one coil deployed. On day 4, after clopidogrel loading, two PEDs were deployed across the aneurysm neck. CTA on day 7 demonstrated complete aneurysm occlusion. The patient's headache and eye pain resolved by day 10. At 6 month clinical follow-up the complete third cranial nerve palsy remains, but he is pain free.

\subsubsection{Thirty Electively Booked Cases \\ 1) Baseline neurological status}

28 of these patients were either asymptomatic, or not disabled by their symptoms (modified Rankin Scale, mRS $=0$ or 1 ) pre-operatively. Patients 3 and 33 were disabled secondary to their original poor clinical grade subarachnoid hemorrhage and had mRS scores of 3 and 4 pre-operatively.

\section{2) Previously treated aneurysms}

Twelve patients were treated electively for aneurysm recanalisation or recurrence after prior treatment; 8 had been previously coiled only, 1 coiled and clipped, 1 coiled with stent assistance and clipped, and 2 had been clipped only. Seven had originally presented with target aneurysm rupture and subarachnoid hemorrhage. Two originally presented with cranial nerve palsies.

\section{3) Unruptured aneurysms not previously treated}

18 cases were elective treatment of unruptured and previously untreated aneurysms with PED. Four of these cases presented with cranial nerve palsy.

\subsection{Aneurysms}

Thirty-seven aneurysms were treated in 33 patients. Patient 12 had an unruptured ophthalmic segment aneurysm treated in combination with a previously coiled superior hypophyseal aneurysm arising from the same ICA. In Patient 14, 4 left ICA aneurysms were treated in a single procedure.

Thirty five ICA aneurysms (7 cavernous, 28 intradural), one basilar trunk fusiform/side-wall aneurysm, and 1 vertebral artery intradural dissecting aneurysm were treated. The mean aneurysm size was $9.0 \mathrm{~mm}, 13$ were large ( $\geq 10 \mathrm{~mm}$ ), and 30 were broad based (neck width $\geq 4$ $\mathrm{mm}$ and/or dome to neck ratio $<1.6$ ).

\subsection{Procedural Results}

\subsubsection{PED Constructs}

In 27 patients a single PED was deployed across the 
aneurysm neck. In 6 cases two telescoping PEDs were deployed.

\subsubsection{Coils with PED}

Endosaccular coils were deployed during the same procedure as PED deployment in 5 patients. In 4 cases this was to increase aneurysm stasis.

Coils were used for a more novel reason in Patient 3, who was a 50 -year-old female with a $26 \mathrm{~mm}$ wide-necked carotid-ophthalmic aneurysm that had previously been coiled after poor clinical grade $\mathrm{SAH}$, and re-packed on two occasions. The rapid flow into the wide neck made navigation of the Marksman microcatheter past the aneurysm neck difficult. Endosaccular coils were deployed which successfully prevented the Marksman microcatheter from prolapsing into the aneurysm sac. See Figure 2.

\subsubsection{PED Deployment}

Deployment of the PED construct was achieved in all cases.

However, noteworthy difficulties were encountered in two cases:

In Patient 3, two PEDs were deployed across the wide aneurysm neck. The distal bumper of the second (proximal) PED delivery wire became trapped at the distal end of the first (distal) PED. Manipulation of the delivery wire resulted in the distal bumper detaching but remaining firmly adhered to the PED. No embolic or ischemic complications were detected on imaging and the patient suffered no clinical adverse event. On Digital Subtraction

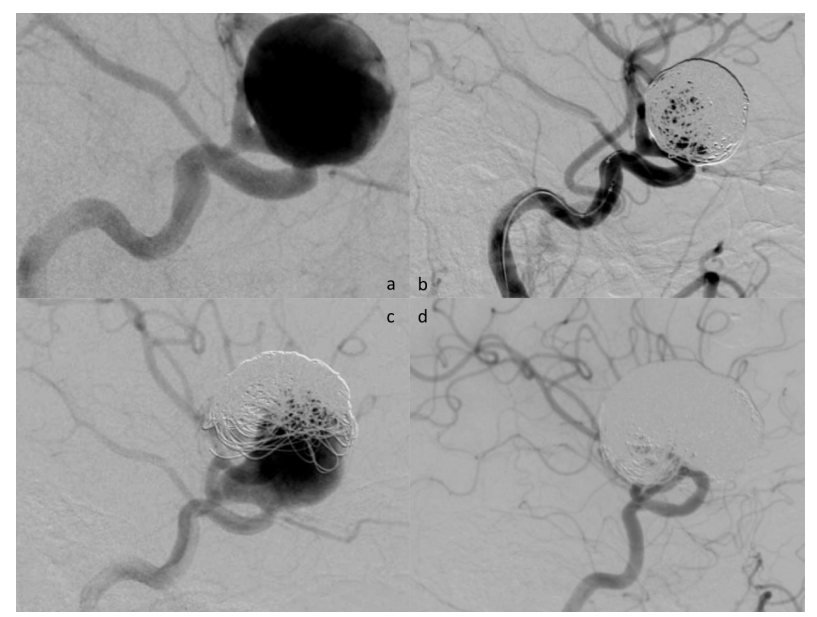

Figure 2. All images are digital subtraction angiography, right internal carotid artery injection, in Patient 3. a) Day 0. A giant ICA aneurysm presented with WFNS grade 5 subarachnoid hemorrhage; b) This was coiled on day 0; c) at 24 months significant coil compaction and recanalization occurred despite 2 elective repeat coilings and unsuccessful surgical exploration; d) 6 months after deployment of a dual PED construct, the aneurysm is completely occluded and the coil mass is collapsing toward the parent ICA.
Angiography (DSA) at 6 months the detached bumper has not moved.

Patient 6 was a 33-year-old female with an ophthalmic segment ICA aneurysm that had recurred after neurosurgical clipping and endovascular stent assisted coiling. During PED deployment the device failed to self-expand in its middle section, creating an hourglass configuration that temporarily obstructed forward flow in the parent ICA. This was remedied with balloon angioplasty, which successfully opened the PED and restored normal ICA flow. The patient was therapeutically anticoagulated throughout the procedure, had cross-flow via patent anterior and posterior communicating arteries, had no ischemic lesion on post-procedure imaging, and suffered no clinical adverse event.

\subsubsection{Immediate Angiographic Results}

Varying degrees of sluggish emptying of contrast from the aneurysm were observed at the end of the case. A "jet" like appearance of aneurysm filling was not demonstrated at the completion of any procedure.

\subsubsection{Repeat Treatments}

Three patients had repeat treatments:

Patient 5 was a 58-year-old male with an unruptured, previously untreated $5 \mathrm{~mm}$ wide necked paraclinoid ICA aneurysm that was treated with a single PED. At 9 months the aneurysm was still filling and a second PED was deployed at 10 months. Three months after the second treatment aneurysm occlusion was demonstrated on DSA.

In Patient 16, a 76-year-old female, a single PED was used to electively treat recanalisation of a previously coiled right posterior communicating artery aneurysm. The patient had originally presented with acute painful third nerve palsy, cured by the original coiling. The day following the PED procedure the patient suffered a new acute painful third cranial nerve palsy. CT and MRI performed to investigate this demonstrated a small punctate focus of intraparenchymal hemorrhage in the ipsilateral temporal lobe. She had no symptoms attributable to this lesion. Due to concern that the nerve palsy represented pressurization of the aneurysm, two more PEDs were deployed on day 2. Aneurysm emptying was much slower and the painful third nerve palsy resolved within 48 hours.

Patient 7 was a 64-year-old female with persistent filling of a wide-necked supraclinoid ICA aneurysm one year after PED treatment. At 13 months a second PED was deployed, and 4 months later the aneurysm is still filling.

\subsubsection{Complications}

No deaths have occurred; however, five patients (15\%) 
suffered transient neurological complications: 2 transient ischemic attacks, 1 intra-procedural SAH, 1 delayed $\mathrm{SAH}$, and 1 exacerbation of mass effect. 2 patients (6\%) suffered permanent neurological complications; 1 delayed stroke and 1 mass effect exacerbation causing permanent ophthalmoplegia.

A clinically silent complication was documented on imaging in 1 patient (3\%), and a single patient (3\%) suffered an asymptomatic femoral artery complication.

\subsubsection{Procedural Complications}

Intra-procedural Aneurysm Rupture and SAH:

Intra-procedural aneurysm rupture occurred during adjunctive aneurysm coiling in patient 15 , discussed above. Other than headache there was no clinical sequel.

\subsubsection{Clinical Complications}

1) Exacerbation of mass effect causing cranial nerve palsies (2 patients):

Patient 16, discussed above, had a new painful third cranial nerve palsy on day 1 post PED treatment. Deployment of two more PEDs occluded the aneurysm and cured the palsy.

Patient 23 was a 41-year-old female who suffered painful third, fourth, sixth, and fifth cranial nerve palsies after treatment of a giant cavernous ICA aneurysm with a PED construct. The aneurysm had initially presented with intermittent ophthalmoplegia. Her new symptoms began 24 hours after the PED deployment, and a CTA performed on the same day demonstrated occlusion of the treated aneurysm. This chronology suggests that the aneurysm thrombosis induced the new cranial nerve palsies. At one year the pain has resolved and the ophthalmoplegia is improving. She is independent and back at work. Her modified Rankin score is 1.

2) Transient ischemic attacks (2 patients):

Two patients had transient neurological deficits within 24 hours of PED deployment. Patient 4 was a 72-yearold female with a wide necked cavernous ICA aneurysm causing a third cranial nerve palsy. 8 hours after two PEDs were deployed, she developed arm weakness and was treated with intra-arterial and intravenous Abciximab.

Angiography demonstrated aneurysm occlusion and no detectable branch occlusion. The weakness resolved completely, with no infarct detected on MRI.

In Patient 3 discussed above, her arm weakness occurred in the first 24 hours and resolved spontaneously.

3) Stroke:

Patient 30 was a 65-year-old female with an unruptured $12 \mathrm{~mm}$ irregular basilar fusiform/side-wall aneurysm. The first PED shortened more than anticipated and did not cover the length of the aneurysm, so a second PED was deployed with approximately $10 \mathrm{~mm}$ of over- lap between the two devices. Her perioperative in-hospital course was uneventful and she was asymptomatic at discharge on day 3 . On day 11 she represented to hospital with left sided weakness and an MRI demonstrated bilateral pontine infarcts. The aneurysm had almost entirely thrombosed. The chronology of the stroke suggests that the affected pontine perforating arteries occluded when the aneurysm thrombosed. At one year she remains mildly disabled with lower limb weakness and ataxia. Her modified Rankin score is 2. Her imaging is demonstrated in Figures 3 and 4.

4) Delayed SAH:

Patient 7, a 64-year-old woman, represented to hospital with headache and nausea 3 days after elective PED treatment of a wide necked unruptured $11 \mathrm{~mm}$ ICA aneurysm. A non-contrast brain CT and an MRI demonstrated a small focus of SAH in a parietal sulcus, ipsilateral but remote from the aneurysm site. The patient's symptoms resolved uneventfully, and she remains asymptomatic at 6 month follow-up. See Figure 5.

5) Asymptomatic Intraparenchymal hemorrhage:

Patient 16, discussed above, had an asymptomatic punctate contralateral temporal lobe hemorrhage demonstrated on CT and MRI on day 1 post PED treatment. See Figure 5.

6) Lower limb complications:

In Patient 15, discussed above, an asymptomatic short segment common femoral artery occlusion was demonstrated on ultrasound at day 35 . This has remained asymptomatic at 6-month follow-up.

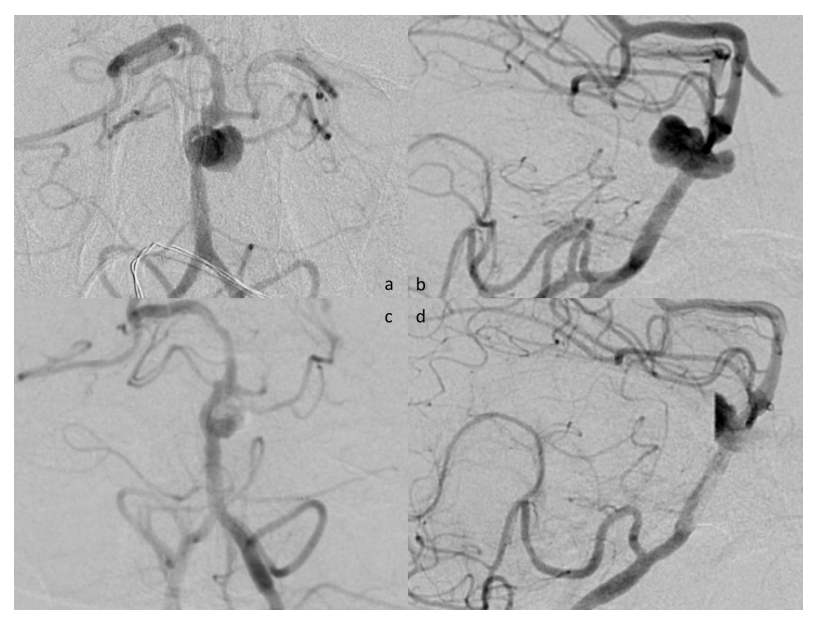

Figure 3. All images are digital subtraction angiography, vertebral artery injection, in Patient 30. a) AP and b) lateral projection demonstrating an an unruptured $12 \mathrm{~mm}$ irregular basilar fusiform/side-wall aneurysm; c) AP and d) lateral images demonstrating contrast stagnation after placement of two PEDs. The first PED shortened more than anticipated and did not cover the length of the aneurysm, so a second PED was deployed with approximately $10 \mathrm{~mm}$ of overlap between the two devices. Her perioperative in-hospital course was uneventful and she was asymptomatic at discharge on day 3 . 


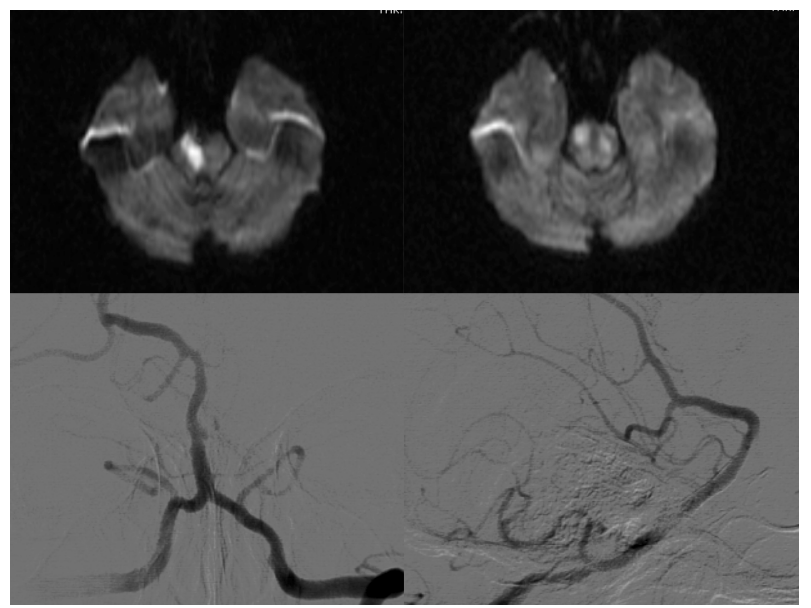

Figure 4. Patient 30 (continued from Figure 3). On day 11 she represented to hospital with left sided weakness. a) and b) axial diffusion weighted MR images demonstrating bilateral pontine infarcts; c) AP and d) lateral digital subtraction angiograms demonstrated that the aneurysm had almost entirely thrombosed. The chronology of the stroke suggests that the affected pontine perforating arteries occluded when the aneurysm thrombosed. At one year she remains mildly disabled with lower limb weakness and ataxia. Her modified Rankin score is 2.

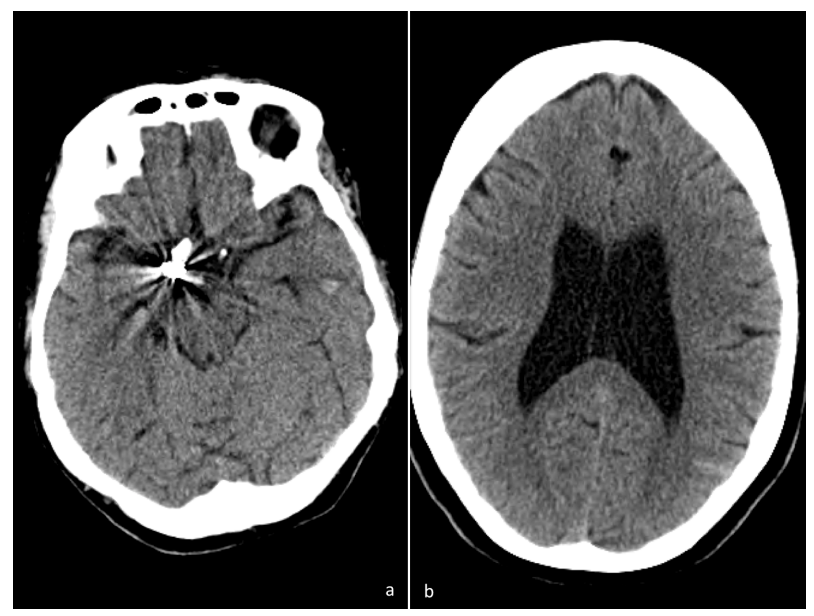

Figure 5. Both images are axial non-contrast brain CT studies, in different patients. a) Patient 16 had an asymptomatic punctate contralateral temporal lobe hemorrhage demonstrated on CT and MRI on day 1 post PED treatment; b) Patient 7, a 64-year-old woman, represented to hospital with headache and nausea 3 days after elective PED treatment of a wide necked unruptured $11 \mathrm{~mm}$ ICA aneurysm. A non-contrast brain CT demonstrated a small focus of SAH in a parietal sulcus, ipsilateral but remote from the aneurysm site. The patient's symptoms resolved uneventfully, and she remains asymptomatic at 6-month follow-up.

\subsection{Imaging Follow-Up Results}

\subsubsection{Imaging Modalities}

All patients have had a minimum of 6 month follow imaging. 28 patients had follow-up angiograms, and 5 pa- tients have been followed up with a combination of CTA and MRA without catheter angiography.

\subsubsection{In-Construct Intimal Hyperplasia}

Patient 32 was a 44-year-old female with an enlarging neck remnant associated with a previously coiled superior hypophyseal aneurysm, originally presenting with SAH. Angiography 6 months after treatment with a single PED construct demonstrated aneurysm occlusion and mild in-construct intimal hyperplasia, with $<25 \%$ stenosis. Her clopidogrel was ceased and her aspirin continued.

\subsubsection{Aneurysm Occlusion}

In 28 patients (85\%) occlusion of the treated aneurysm(s) has been demonstrated. Of these, 6 (21\%) were demonstrated at less than 30 days, $16(57 \%)$ at less than 6 months, and 25 (89\%) within one year. In 3 cases (11\%) aneurysm occlusion was first demonstrated at greater than one year post treatment.

In two of these cases (Patient 9 and Patient 16) this was the earliest angiogram post PED treatment, due to patient choice. Patient 5, discussed above, was re-treated with a second PED on day 315 and occlusion was then demonstrated n day 396 after the first PED treatment.

\subsubsection{Persistent Aneurysm Filling}

The neck remnant of the ophthalmic segment ICA aneurysm treated with 2 PEDs in Patient 6, discussed above, had reduced in size and significantly slower endosaccular flow was demonstrated at 7 months and 16 months. This aneurysm had been previously treated with Neuroform (Stryker Neurovascular, California) stent assisted coiling, which has been previously reported to reduce the incident of aneurysm closure [3,6,7,15]. A patent ophthalmic artery was still also filling from the aneurysm.

Patient 7 is discussed above.

Patient 12 was a 40-year-old female treated electively with a single PED for two aneurysms arising from the left ICA. On day 211 the previously untreated ophthalmic segment aneurysm had occluded, and the recanalised neck of the previously coiled superior hypophyseal aneurysm had reduced in size with the coil mass collapsing down towards the PED.

In Patient 17, a 72-year-old female, an unruptured $16 \mathrm{~mm}$ cavernous segment ICA aneurysm causing cranial nerve symptoms was treated with a single PED. At 6 months the aneurysm has reduced in size to $6 \mathrm{~mm}$ and the cranial nerve symptoms have resolved.

Patient 22 was a 68-year-old female in whom a single PED was used to treat an unruptured wide necked $8 \mathrm{~mm}$ cavernous ICA aneurysm that presented with cranial nerve palsy. At 1 year the aneurysm is not occluded and follow-up imaging is planned for 2 years post treatment. 


\subsection{Clinical Follow-Up Results}

All patients have had a minimum of 12 months clinical follow-up. Mean follow-up is 21 months (range 13 to 32 months). No deaths have occurred. The clinical status and modified Rankin Score (mRS) has not changed from its preoperative state in 31 cases (94\%).

Patient 23, discussed above, has persistent ophthalmoplegia which is not disabling and she is back at work, her mRS equals 1 .

Patient 30 remains disabled by her pontine stroke, but is independent. Her mRS equals 2.

\section{DISCUSSION}

1) PED deployment:

As the collective operator experience increases, the deployment technique has evolved from a predominantly "unsheathing" method as used for most other stents, to a more balanced "push and pull" method with more forward load on the PED delivery device. Anecdotally this increases the wall apposition and distal anchorage of the PED, reducing the incidence of the device slipping proximally during the unsheathing of its remainder. We observed this in our series and this is also reported by authors in other recent series $[6,16]$.

2) Re-capturing the distal bumper:

After the PED is released, the microcatheter is re-advanced over the delivery wire (inside the PED lumen) and the distal capture coil or bumper. This bumper has a larger diameter than the delivery wire and can be difficult to recapture when the system is in a tortuous segment. In a single case in our series the distal end of the delivery wire broke off during repeated attempts to recapture it. This remained stationary within the PED construct at follow-up and caused no clinical sequel. Other authors have reported problems recapturing the distal bumper in as many as $26 \%$ of cases $[6,16]$.

3) Use of balloon angioplasty to remodel the PED once deployed:

The use of balloons such as the Hyperglide or Hyperform (ev3, Irvine) to assist the PED in opening completely and apposing the endothelial surface has become commonplace, and is described by many authors [2,5-9, 17-19]. Fischer et al. report using this technique in 13 (15\%) of 88 patients, and the Pipeline for Uncoilable or Failed Aneurysms (PUFS) trial data records this in 18 (16\%) of 111 patients [5,6]. Fischer et al. also report the use of angioplasty to correct parent artery stenosis before PED deployment [6]. The technique is not without risk, one patient in the pipeline embolization device for the Intracranial Treatment of Aneurysms (PITA) trial suffered an iatrogenic ICA rupture during balloon angioplasty to correct a stenosis after PED deployment [4].

The successful use of endosaccular coils to prevent prolapse of the Marksman microcatheter into a giant aneurysm sac (Patient 3) has not previously been described.

4) Aneurysm Occlusion:

Our overall rate of demonstrated aneurysm occlusion was $85 \%$ which is similar to the $84 \%$ recorded in the PUFS trial [5], and the $85 \%$ recorded by McAuliffe et al. [8]. These closure rates are less than the $93 \%$ - 94\% occlusion rates demonstrated in the early series by Lylyk et al., Szikora et al., and the PITA trial [2-4]; but greater than the $74 \%$ in the largest published PED series to date [6].

These rates compare favorably with stent assisted coiling techniques. Large series of patients treated with laser cut scaffolding type stents record rates of $24 \%$ $28 \%$ incomplete aneurysm occlusion. [20,21] A series of 19 patients treated with a more advanced Y-stenting technique for basilar tip aneurysms recorded aneurysm closure in $63 \%$ [22].

5) Repeat Treatments:

Three (9\%) of 33 patients in our series had repeat PED treatments, in one case to treat recurrent mass effect after an elective single PED treatment of aneurysm recanalisation, and in two cases to treat persistent aneurysm filling at 9 and 13 months after single PED treatment. Fischer et al. also recorded a $9 \%$ retreatment rate in their series [6].The largest series of stent assisted coiling with medium term follow-up (using the Neuroform stent) reported a recanalisation rate of $28 \%$ and a retreatment rate of $15 \%$ [20].

6) Thromboembolic and Ischemic complications:

Two patients (6\%) suffered transient ischemic attacks. One patient (3\%) suffered a stroke, a pontine infarction that has resulted in mild/moderate disability, with preserved independence. In published series of greater than 10 cases; rates of TIAs range from $0 \%$ to $17 \%$, and strokes from $0 \%$ to $17 \%[1-8,15,16,23,24]$.

The single permanent neurological deficit in our was due to a basilar perforator infarction at the site of an overlapping double PED construct. The vertebrobasilar system differs embryologically from the anterior circulation, and its supply to the rhombencephalon and mesencephalon is via perforators arising directly from the parent artery [25]. Occlusion of a single perforator can, as in this case, cause bilateral and disabling brainstem infarction.

The majority of the cases published in the literature involve PEDs deployed in the ICA, however posterior circulation PED cases provide a relatively greater share of the ischemic complications $[6,8,15,16]$. Twenty two (25\%) of the 88 patients in the series by Fischer et al. had posterior circulation aneurysms treated with PED. Of the 5 serious complications, $3(60 \%)$ were in basilar aneurysm cases [6]. A silent pontine infarct was reported 
in a basilar artery PED case by Martin et al. [19]. Recent case reports have documented three very late PED construct occlusions in posterior circulation cases, 2 of them fatal [26,27]. A case report of an A1 perforator infarction after overlapping PEDs is also reported [28].

Large series of patients treated with laser cut scaffolding type stents record rates of 5\% - 7\% permanent neurological complications [20,21]. A series of 19 patients treated with Y-stenting technique for basilar tip aneurysms recorded initial complications of $32 \%$, and delayed thromboembolic complications in 11 [22].

7) Hemorrhagic complications:

One case of very small volume parietal sulcal SAH at day 3 post ipsilateral ICA PED presented with headache and nausea which resolved uneventfully. One case of asymptomatic punctate temporal lobe hemorrhage on day 1 post ipsilateral ICA PED was recorded.

Intraparenchymal and non-aneurysmal SAH in the territory supplied by the parent artery (“dependent brain”) are emerging as complications that are possibly peculiar to flow diverters.

In the PUFS trial [4], intracerebral hemorrhages have been recorded in 111 cases [5]. Fischer et al. report 3 dependent brain hemorrhages in 88 cases [6], Cruz et al. reported 4 intraparenchymal hemorrhages in a series of 66.10 and single cases have been reported by several other authors $[7,9,16]$.

In the largest series of stent assisted coiling with medium term follow-up, 19 peri-procedural strokes were recorded in 284 patients (6.7\%). Five deaths (1.8\%) were associated with new infarctions. Five $(1.8 \%)$ procedural perforations resulted in a new SAH. No dependent brain intraparenchymal hemorrhages were recorded, despite a similar regimen of dual antiplatelet pre-medication and peri-procedural heparinisation. No delayed aneurysm ruptures were encountered in this study.

8) Increased Mass Effect after PED:

Of the 7 patients who had initially presented with aneurysm mass effect, 2 (29\%) suffered worsening oculomotor dysfunction associated with increased aneurysm mass effect post PED treatment. This is similar to the rates published by Lylyk et al. (3 of 4, 75\%), McAuliffe et al. (3 of 16, 19\%), and the PUFS trial (9 of 111, 8\%) $[3,5,8]$.

9) The use of PED in acute SAH:

The single acute SAH case in this series was complicated by intra-procedural aneurysm rupture during concomitant endosaccular coiling, without any permanent deficit, and a full neurological recovery. Published data with larger numbers of acutely ruptured aneurysms treated with PED caution that the technique should only be used if there is no other option, and that endosaccular coils should be deployed when possible [7,9,15,19,24].

10) In-construct stenosis:
In-construct stenosis was only recorded in one patient which is at the lower range of published incidence $[1,3,5,8,15]$.

11) Treatment of aneurysmal carotid-cavernous fistula with PED:

The use of a PED construct to treat a direct carotid-cavernous fistula (CCF) is a novel technique that has previously been described for treatment of a traumatic CCF [29], but not when secondary to aneurysm rupture, as was the case in Patient 13.

12) Long-term clinical follow-up:

At current follow-up (mean 21, range 13 - 32 months), $2(6 \%)$ of 33 patients have a neurological status that is worse than before their PED treatment; due to increased mass effect causing ophthalmoplegia (mRS 1), and pontine infarction (mRS 2) respectively. Both of these events occurred in the short term at 24-hours and 11 days post PED treatment. No late complications and no deaths have occurred.

Our minimum length of clinical follow-up (13 months) in 33 patients is the longest of any published series of greater than 20. Smaller series by Szikora et al. and Deutschmann, et al. of 18 and 12 cases respectively have also published 12-month clinical results [1,2]. The PUFS trial recorded 12 month clinical follow-up for 92 (85.2\%) of 111 cases in the publicly accessible FDA documents, but these only record itemized numbers of clinical events without any explanation or discussion [5].

13) Other flow diverters:

The SILK ${ }^{\mathrm{TM}}$ flow diverter (SFD, BALT Extrusion, Paris) is the other flow diverting construct currently available. Its concept is similar, however reports of delayed aneurysm rupture suggest a different safety profile to the PED [30-32].

Early SILK cases series also report higher overall complication rates than the equivalent early PED series. [3,8,33-37].

As well, MicroVention-TERUMO (Tustin, CA, USA) has recently introduced FRED ${ }^{\mathrm{TM}}$ (Flow Re-Direction Endoluminal Device), with the clinical trials and case series are underwent.

\section{CONCLUSION}

The technique of flow diversion and endoluminal reconstruction differs greatly from the established endosaccular packing techniques of standard coiling, balloon remodeling, and stent assisted coiling. This is reflected in the different technical issues at deployment, aneurysm closure rates, and the spectrum of complications. As well as being associated with higher rates of aneurysm closure and lower rates of retreatment, PED is associated with an altered spectrum of complications relative to other techniques. The rates of clinically significant complications compare favorably with published data on stent assisted 
coiling. This study of 33 patients is the first of its size to present greater than 12-month clinical follow-up and analysis of complications.

\section{CONFLICT OF INTEREST STATEMENT}

We declare that we have no conflict of interest.

\section{ACKNOWLEDGEMENTS}

Ms Carolina Radwan for her assistance with database intenance.

\section{REFERENCES}

[1] Deutschmann, H.A., Wehrschuetz, M., Augustin, M., et al. (2011) Long-term follow-up after treatment of intracranial aneurysms with the pipeline embolization device: Results from a Single Center. American Journal of Neuroradiology.

[2] Szikora, I., Berentei, Z., Kulcsar, Z., et al. (2010) Treatment of intracranial aneurysms by functional reconstruction of the parent artery: The Budapest experience with the pipeline embolization device. American Journal of Neuroradiology, 31, 1139-1147. http://dx.doi.org/10.3174/ajnr.A2023

[3] Lylyk, P., Miranda, C., Ceratto, R., et al. (2009) Curative endovascular reconstruction of cerebral aneurysms with the pipeline embolization device: The Buenos Aires experience. Neurosurgery, 64, 632-642. http://dx.doi.org/10.1227/01.NEU.0000339109.98070.65

[4] Nelson, P.K., Lylyk, P., Szikora, I., et al. (2011) The pipeline embolization device for the intracranial treatment of aneurysms trial. American Journal of Neuroradiology, 32, 34-40.

[5] FDA. (2011) Summary of safety and effectiveness data. pipeline embolization device.

[6] Fischer, S., Vajda, Z., Aguilar Perez, M., et al. (2011) pipeline embolization device (PED) for neurovascular reconstruction: initial experience in the treatment of 101 intracranial aneurysms and dissections. Neuroradiology, 54, 369-382.

[7] McAuliffe, W. and Wenderoth, J.D. (2011) Immediate and midterm results following treatment of recently ruptured intracranial aneurysms with the pipeline embolization device. American Journal of Neuroradiology, 33, 487-493.

[8] McAuliffe, W., Wycoco, V., Rice, H., et al. (2012) Immediate and Midterm Results following Treatment of Unruptured Intracranial Aneurysms with the pipeline embolization device. American Journal of Neuroradiology, 33, 164-170. http://dx.doi.org/10.3174/ajnr.A2727

[9] Velat, G.J., Fargen, K.M., Lawson, M.F., et al. (2011) Delayed intraparenchymal hemorrhage following pipeline embolization device treatment for a giant recanalized ophthalmic aneurysm. Journal of NeuroInterventional Surgery.

[10] Cruz, J.P., Chow, M., O’Kelly, C., et al. (2012) Delayed ipsilateral parenchymal hemorrhage following flow di- version for the treatment of anterior circulation aneurysms. American Journal of Neuroradiology, 33, 603-608. http://dx.doi.org/10.3174/ajnr.A3065

[11] Kallmes, D.F., Ding, Y.H., Dai, D., et al. (2009) A second-generation, endoluminal, flow-disrupting device for treatment of saccular aneurysms. American Journal of Neuroradiology, 30, 1153-1158. http://dx.doi.org/10.3174/ajnr.A1530

[12] Fiorella, D., Lylyk, P., Szikora, I., et al. (2009) Curative cerebrovascular reconstruction with the pipeline embolization device: The emergence of definitive endovascular therapy for intracranial aneurysms. Journal of NeuroInterventional Surgery, 1, 56-65.

http://dx.doi.org/10.1136/jnis.2009.000083

[13] Fiorella, D., Woo, H.H., Albuquerque, F.C., et al. (2008) Definitive reconstruction of circumferential, fusiform intracranial aneurysms with the pipeline embolization device. Neurosurgery, 62, 1115-1121. http://dx.doi.org/10.1227/01.neu.0000325873.44881.6e

[14] Oncel, D., Oncel, G., Karaca, M. (2007) Coronary stent patency and in-stent restenosis: Determination with 64section multidetector CT coronary angiography-Initial Experience1. Radiology, 242, 403-409. http://dx.doi.org/10.1148/radiol.2422060065

[15] de Barros Faria, M., Castro, R.N., Lundquist, J., et al. (2011) The role of the pipeline embolization device for the treatment of dissecting intracranial aneurysms. American Journal of Neuroradiology. http://dx.doi.org/10.3174/ajnr.A2671

[16] Lubicz, B., Collignon, L., Raphaeli, G., et al. (2011) Pipeline flow-diverter stent for endovascular treatment of intracranial aneurysms: Preliminary experience in 20 patients with 27 aneurysms. World Neurosurgery, 76, 114119. http://dx.doi.org/10.1016/j.wneu.2011.02.015

[17] Aurboonyawat, T., Schmidt, P.J., Piotin, M., et al. (2011) A study of the first-generation pipeline embolization device morphology using intraoperative angiographic computed tomography (ACT). Neuroradiology, 53, 23-30. http://dx.doi.org/10.1007/s00234-010-0709-2

[18] Hauck, E.F., Natarajan, S.K., Langer, D.J., et al. (2010) Retrograde trans-posterior communicating artery snareassisted rescue of lost access to a foreshortened pipeline embolization device: Complication management. Neurosurgery, 67, 495-502. http://dx.doi.org/10.1227/NEU.0b013e3181f8530d

[19] Martin, A.R., Pablo Cruz, J., Matouk, C.C., et al. (2011) The Pipeline flow-diverting stent for exclusion of ruptured intracranial aneurysms with difficult morphologies. Neurosurgery, 70, 21-28.

[20] Fiorella, D., Albuquerque, F.C., Woo, H., et al. (2010) Neuroform stent assisted aneurysm treatment: Evolving treatment strategies, complications and results of longterm follow-up. Journal of NeuroInterventional Surgery, 2, 16-22. http://dx.doi.org/10.1136/jnis.2009.000521

[21] Mocco, J., Snyder, K.V., Albuquerque, F.C., et al. (2009) Treatment of intracranial aneurysms with the Enterprise stent: a multicenter registry. Journal of Neurosurgery, 110, 35-39. http://dx.doi.org/10.3171/2008.7.JNS08322 
[22] Spiotta, A.M., Gupta, R., Fiorella, D., et al. (2011) Midterm results of endovascular coiling of wide-necked aneurysms using double stents in a Y configuration. Neurosurgery, 69, 421.

http://dx.doi.org/10.1227/NEU.0b013e318214abbd

[23] Puffer, R.C., Kallmes, D.F., Cloft, H.J. and Lanzino, G. (2012) Patency of the ophthalmic artery after flow diversion treatment of paraclinoid aneurysms. Journal of Neurosurgery, 116, 892-896.

[24] Pistocchi, S., Blanc, R., Bartolini, B., et al. (2012) Flow diverters at and beyond the level of the circle of willis for the treatment of intracranial aneurysms. Stroke, 43, 10321038.

http://dx.doi.org/10.1161/STROKEAHA.111.636019

[25] Charles, R. (2010) Normal and abnormal embryology and development of the intracranial vascular system. Neurosurgery Clinics of North America, 21, 399-426. http://dx.doi.org/10.1016/j.nec.2010.03.011

[26] Klisch, J., Turk, A., Turner, R., et al. (2011) Very late thrombosis of flow-diverting constructs after the treatment of large fusiform posterior circulation aneurysms. American Journal of Neuroradiology, 32, 627-632. http://dx.doi.org/10.3174/ajnr.A2571

[27] Fiorella, D., Hsu, D., Woo, H.H., et al. (2010) Very late thrombosis of a pipeline embolization device construct: Case report. Neurosurgery, 67, onsE313-314; discussion onsE314.

[28] van Rooij, W.J. and Sluzewski, M. (2010) Perforator infarction after placement of a pipeline flow-diverting stent for an unruptured A1 aneurysm. American Journal of Neuroradiology, 31, E43-E44. http://dx.doi.org/10.3174/ajnr.A2034

[29] Nadarajah, M., Power, M., Barry, B., et al. (2011) Treatment of a traumatic carotid, cavernous fistula by the sole use of a flow diverting stent. Journal of NeuroInterventional Surgery.

[30] Turowski, B., Macht, S., Kulcsar, Z., et al. (2011) Early fatal hemorrhage after endovascular cerebral aneurysm treatment with a flow diverter (SILK-Stent): Do we need to rethink our concepts? Neuroradiology, 53, 37-41. http://dx.doi.org/10.1007/s00234-010-0676-7

[31] Kulcsar, Z., Houdart, E., Bonafe, A., et al. (2011) Intra-aneurysmal thrombosis as a possible cause of delayed aneurysm rupture after flow-diversion treatment. American Journal of Neuroradiology, 32, 20-25.

[32] Cloft, H.J. (2011) Flow diversion for cerebral aneurysms: A cautionary tale. American Journal of Neuroradiology, 32, 26.

[33] Szikora, I., Berentei, Z., Kulcsar, Z., et al. (2010) Treatment of intracranial aneurysms by functional reconstruction of the parent artery: The budapest experience with the pipeline embolization device. American Journal of Neuroradiology, 31, 1139-1147.

[34] McAuliffe, W., Wycoco, V., Rice, H., et al. (2011) Immediate and midterm results following treatment of unruptured intracranial aneurysms with the pipeline embolization device. American Journal of Neuroradiology, 33, 164-170.

[35] Lubicz, B., Collignon, L., Raphaeli, G., et al. (2010) Flow-diverter stent for the endovascular treatment of intracranial aneurysms: A prospective study in 29 patients with 34 aneurysms. Stroke, 41, 2247-2253. http://dx.doi.org/10.1161/STROKEAHA.110.589911

[36] Kulcsar, Z., Ernemann, U., Wetzel, S.G., et al. (2010) High-profile flow diverter (silk) implantation in the basilar artery: Efficacy in the treatment of aneurysms and the role of the perforators. Stroke, 41, 1690-1696. http://dx.doi.org/10.1161/STROKEAHA.110.580308

[37] Byrne, J.V., Beltechi, R., Yarnold, J.A., et al. (2010) Early experience in the treatment of intra-cranial aneurysms by endovascular flow diversion: A multicentre prospective study. PLoS ONE, 5, e12492. http://dx.doi.org/10.1371/journal.pone.0012492 